\title{
Derecho de Capacitación y desempeño laboral en la Dirección Regional Agraria. Caso: Amazonas
}

\author{
Training and labor performance law in the Regional Agricultural Directorate. \\ Case: Amazonas
}

\section{AUTOR 1: \\ Armando Edgardo Figueroa Sánchez \\ Afiliación: Universidad San Martín de Porres, Lima-Perú / Instituto de Gobierno y de Gestión Pública. Doctor en \\ Planificación y Gestión. Docente de Maestría, y Doctorado, Lima-Perú. \\ ORCID: 0000-0003- 1891-9739}

Correo:

afigueroas@usmp.pe

\section{AUTOR 2:}

\section{Dr. Iván Hidalgo} Romero

Afiliación: : Universidad San Martín de Porres, Lima-Perú / Instituto de Gobierno y de Gestión Publica. Doctor en Gobierno y Política Pública. Director (e) Instituto de Gobierno y de Gestión Pública

ORCID: 0000-00016502-3738

Correo:

ihidalgor@usmp.pe

\section{AUTOR 3:}

Mg. Jhon Stalyn Maco Tuesta

Afiliación: Universidad Nacional Intercultural Fabiola Salazar Leguía de Bagua. Lima-Perú. Maestro en Gestión Pública, Lima - Perú. ORCID: 0000-00020110-6443

Correo: stalmat@gmail.com
Recibido: 17 de julio de 2019

Aprobado: 02 de noviembre de 2020

\section{Resumen:}

Se presentó como objetivo, determinar cómo las actividades de capacitación están relacionadas al rendimiento de los servidores de la de las oficinas del Ministerio de Agricultura de Amazonas, en el año 2019. Fue una investigación, descriptiva-relacional. Se estableció una muestra de 73 trabajadores, y 16 jefes directos de los servidores. Se encontró que, acerca de la planificación del adiestramiento y rendimiento de los trabajadores, se encontró una asociación, entre estas variables, encontrándose correlación de 0,50. También existe asociación con la ejecución del adiestramiento y el promedio del rendimiento, encontrándose una correlación de 0,58. Todo esto indica que, a pesar de que los trabajadores no reciben un buen adiestramiento, su desempeño laboral también es regular. El $62 \%$ de los trabajadores expresaron estar de acuerdo que, las capacitaciones contribuyen en el desempeño de los servidores, presentando similitud con (Mayurí, 2008), quien encontró que, el $60 \%$ de los trabajadores, indicó que siempre, la capacitación podría incrementar el desempeño de los trabajadores.

Palabras clave: capacitación, planificación, ejecución, desempeño.

\section{Abstract:}

The objective was to determine how the training activities are related to the performance of the servers of the offices of the Ministry of Agriculture of Amazonas, in the year 2019. It was a descriptive-relational investigation. A sample of 73 workers and 16 direct chiefs of the servers were established. It was found that, regarding the planning of the training and performance of the workers, an association was found, among these variables, finding a correlation of 0.50 . There is also an association with the execution of training and the average performance, finding a correlation of 0.58 . All this indicates that, although workers do not receive good training, their job performance is also regular. $62 \%$ of the workers expressed their agreement that the training contributes to the performance of the servers, presenting a similarity with (Mayurí, 2008), who found that $60 \%$ of the workers indicated that the training could always increase The performance of the workers.

Keywords: training, planning, execution, performance. 


\section{INTRODUCCIÓN}

Últimamente el desarrollo de procesos en los gobiernos, y empresas, está promoviendo el uso de procedimientos o estrategias para una mejor gestión, de los recursos institucionales. Una de ellas es la evaluación de cómo se vienen desempeñando los trabajadores, especialmente en el sector distrital para (Bonnefoy, 2005, pág. 9).

En Perú se crea la (Autoridad Nacional del Servicio Civil, 2019), por DL. № 1023, institución pública que rige los recursos humanos, de todos los órganos del estado, institución que entre otras funciones pretende incrementar el rendimiento de los trabajadores públicos, procurando la profesionalización, identificando méritos, y estableciendo reglas con deberes, y derechos.

Es importante que: en la administración pública, la exigencia para la efectividad con un buen uso de los recursos, constituyen los elementos base, de los gobiernos en el propósito de lograr mejor desempeño. Por lo tanto, en cuanto los procesos son deficientes, se precisa efectuar un replanteamiento de los procesos, y los subprocesos, como lo señala (Mayurí, 2008, pág. i)

Los procesos de perfeccionamientos, se forman a través de los programas de adiestramiento, los que procuran "cubrir los vacíos identificables en el diagnóstico, y con ello iniciar el proceso de transformación, en el núcleo de la cultura organizacional, a fin de que los cambios introducidos se consoliden y por ende se eleve el rendimiento del sistema empresarial". (Mayurí, 2008, pág. 10).

Es la (Autoridad Nacional del Servicio Civil -SERVIR, 2019), quien plantea a las instituciones del estado peruano presentar el plan de desarrollo de personas (PDP), promoviendo el adiestramiento de los trabajadores del estado.

De acuerdo al (Decreto Supremo № 009-2010-PCM, 2010), en el artículo 3, las instituciones públicas, están obligadas a presentar un PDP. Haciendo una revisión de la continuidad en la presentación de estos planes y desarrollo de personas, se encontró (figura 1) que, el Gobierno Regional de Amazonas, no ha tenido continuidad en la presentación de estos planes. 


\begin{tabular}{|c|c|c|c|c|c|c|c|c|c|c|c|}
\hline & \multirow{2}{*}{ NOMBRE DE LA ENTIDAD } & \multirow{2}{*}{ DEPARTAMENTO } & \multicolumn{9}{|c|}{ PRESENTACIÓN PDP } \\
\hline & & & QUINQUENAL & 2012 & 2013 & 2014 & 2015 & 2016 & 2017 & 2018 & 2019 \\
\hline 1 & $\begin{array}{l}\text { GOBIERNO REGIONAL DE } \\
\text { LIMA }\end{array}$ & LIMA & NO & NO & NO & NO & $\underline{s}$ & $\underline{s}$ & $\underline{S l}$ & $\underline{\text { Sl }}$ & NO \\
\hline 2 & $\begin{array}{l}\text { GOBIERNO REGIONAL DE } \\
\text { AMAZONAS }\end{array}$ & AMAZONAS & $\underline{S l}$ & $\underline{s}$ & $\underline{s}$ & $\underline{\mathrm{Sl}}$ & NO & NO & NO & $\underline{\mathrm{Sl}}$ & NO \\
\hline 3 & $\begin{array}{l}\text { GOBIERNO REGIONAL DE } \\
\text { ANCASH }\end{array}$ & ANCASH & NO & NO & NO & NO & NO & NO & NO & NO & NO \\
\hline
\end{tabular}

Figura 1 Plan desarrollo de Personas, Gobiernos Regionales

Fuente: (Autoridad Nacional del Servicio Civil -SERVIR, 2019)

Objetivo de la investigación. Determinar cómo las actividades de capacitación están relacionadas al desempeño de los servidores de la Dirección Regional Agraria Amazonas, del año 2019. Como antecedentes internacionales, en México, el estudio de (Días, 2011, pág. 4), resultó: la autoevaluación del adiestramiento, influye en el rendimiento laboral. En la investigación de por (Alvarado, Gonzalez, Lopez, \& Perez, 2002, pág. 60), encontró que, "El 93.33\% de los empleados, consideran que los contenidos de la última capacitación estaban acordes a las necesidades de su puesto, sólo el $6.67 \%$ considera que no están acorde los contenidos". Por otro lado, (Ramírez, 2014, pág. 64), en México quien encontró que: la capacitación de los trabajadores, podría predecir su desempeño, señalando que, una variable influye sobre la otra.

Asimismo, (Lacavex, 2013), señala que, gran parte de las instituciones, se encuentran incumpliendo con la constitución de las comisiones mixtas de las capacitaciones y adiestramiento. Por su parte, (Parra-Penagos \& Rodríguez-Fonseca, 2016), establecieron lo siguiente: el adiestramiento influye en la calidad.

Entre los antecedentes nacionales, la investigación de (Fuentes, 2014, pág. 105), entre sus resultados encontró que: para el $32 \%$ de los servidores, cumplen con los objetivos y metas, el 5\% no ha recibido adiestramiento". Por otro lado, (Colque, 2016), en los resultados obtenidos: "El desempeño laboral no es el esperado, en el caso de la responsabilidad, para el $43 \%$ es desfavorable, para el $29 \%$ es favorable, y muy favorable, mientras que para el $28 \%$, la responsabilidad, se desarrolla en nivel medio. Por otro lado, el desempeño laboral no es el esperado, en el caso de la responsabilidad, para el $43 \%$ es desfavorable, para el $29 \%$ es favorable, y muy favorable, mientras que para el $28 \%$, la responsabilidad, se desarrolla en nivel medio". También, (Rengifo, 2018, pág. 57), encontró que, para el 81,5\% la percepción del desempeño es regular, para el 18,5\% es alto (Rengifo, 2018, pág. 77), concluye que, el adiestramiento, se relaciona con el desempeño. Por su parte, (Noda, 2017, pág. 
52), encontró que, el $100 \%$ de los servidores, entre siempre y frecuentemente, alcanzan técnicas facilitándole el trabajo.

Por su parte, (Mayurí, 2008, pág. 75), encontró que, existe relación entre el programa de adiestramiento y el desempeño. Para (Obregón, y otros, 2008), "La capacitación es el conjunto de acciones de preparación, continuas y planificadas, concebido como una inversión, que desarrollan las organizaciones dirigidas a mejorar las competencias y calificaciones de los trabajadores, para cumplir con calidad las funciones del cargo, asegurar su desempeño exitoso y alcanzar los máximos resultados productivos o de servicios." El autor (Jamaica, 2015), señala que son los directivos, quienes deben: "motivar y estimular a los colaboradores a fin de que se puedan preparar, para encarar los cambios que trae el entorno y la globalización como factor de integración comercial, independientemente del tamaño de la organización"

Según (Qian \& Zhou, 2008) en la capacitación se debe tener en cuenta: la participación total; la rentabilidad; y la instrumentación.

La (Autoridad Nacional del Servicio Civil, 2016, pág. 12), establecida por (Decreto Legislativo № 1023, 2008), propicia el derecho a la educación, mediante el adiestramiento de los servidores públicos peruanos, señalando que: es un proceso de tres fases: Planificación, Ejecución y Evaluación. Establece, además, que este proceso, se relaciona con el rendimiento o desempeño. Es esta consideración, entre otras, la que motiva la presente investigación, a fin de determinar cómo se desarrolla la capacitación en la DRA. EI Perú, según la (OIT - Lima, 2019), es su miembro a partir del año 1919, por lo tanto, debe cumplir con el C142 - del Convenio acerca de los recursos humanos de la (Organización Internacional del Trabajo, 1975), mediante la cual, en el art. 1, indica que, los miembros tendrían que, patrocinar y practicar programas y políticas en el entorno de la profesionalización.

Respecto al desempeño laboral, (Chiavenato, 2009), señala que: "La evaluación del desempeño es una valoración, sistemática, de la actuación de cada persona en función de las actividades que desempeña, las metas y los resultados que debe alcanzar, las competencias que ofrece y su potencial de desarrollo".

La Dirección Regional Agraria, es un órgano dependiente del Gobierno Regional Amazonas a través de la Gerencia Regional de Desarrollo Económico. Por lo que, es importante saber lo que piensan los trabajadores sobre los adiestramientos en los que han participado.

Respecto a la estructura del artículo: primeramente, se presenta la introducción, en donde se describe la situación problemática, se exponen los objetivos, y se ostentan los antecedentes, y bases teóricas relacionadas con las variables estudiadas. En segundo lugar, se presenta el método, señalando la metodología utilizada, población, y

Calle. Martín Dulanto 101, Miraflores. Lima 18 - Perú. Teléfonos: 241-4273, 241-4274, 2414275 Anexo 101/106. Correo : jsanchezo1@usmp.pe ISSN: 2414-4991 
muestra, se describen los instrumentos, y el procesamiento de la información. Posterior a esto, se presentan los resultados, relacionados con los servidores de la DRA-Amazonas. Luego, se presenta la discusión, realizando comparaciones de los resultados arrojados, con otros autores. Finalmente se presentan las conclusiones, las mismas que, se desprenden del desarrollo del trabajo.

\section{MÉTODO}

Fue una investigación cuantitativa, no experimental, descriptiva-relacional, transversal. Se contó con dos poblaciones finitas, la primera población, fue de 123 servidores de la Dirección Regional Agraria (DRA) de Amazonas, datos suministrado por la misma institución en referencia al personal nombrado y contratado. La segunda población, estuvo conformada por 16 directivos, y responsables de la DRA, quienes evaluaron el desempeño de los servidores a su cargo. La muestra fue probabilística de 73 trabajadores, quienes evaluaron la capacitación. Asimismo, se utilizó una muestra de 16 directivos, según cada una de sus unidades de trabajo, quienes evaluaron el desempeño. Utilizando la encuesta, como técnica de recolección de información, mediante dos instrumentos denominados cuestionarios, desarrollados de la siguiente manera: El primer cuestionario sobre las capacitaciones, se aplicó a los 73 servidores de la DRA Amazonas, conformado por veinte (20) preguntas, en este instrumento consideró la variable capacitación. En cuanto al segundo cuestionario, relacionado con el desempeño de los servidores, se aplicó a los 16 jefes de departamento, este cuestionario estuvo conformado por doce (12) preguntas, sobre las actividades del adiestramiento y el desempeño de los servidores. Como técnicas de procesamiento y análisis estadístico, se tiene que, para analizar los resultados, fue utilizado el software estadístico SPSS, y MINITAB versión 18, en cumplimiento con los objetivos de la presente investigación.

\section{RESULTADOS.}

1. Actividades de capacitación de los trabajadores de la Dirección Regional Agraria (DRA) Amazonas 2019, en cumplimiento de las disposiciones legales de la (Autoridad Nacional del Servicio Civil, 2019)

1.1 Capacitaciones y su contribución en el desempeño de los trabajadores de la Dirección Regional Agraria

El $62 \%$ de los trabajadores expresaron estar de acuerdo que, las capacitaciones contribuyen en el desempeño de los servidores. El 25\% de los encuestados manifestó estar en desacuerdo, mientras que solo el 13\% se mostró indiferente. Es decir, de acuerdo a los resultados, el $38 \%$ de los trabajadores, no está conforme con lo 
establecido por la normatividad legal de (Autoridad Nacional del Servicio Civil SERVIR, 2019)

Tabla 1. Capacitaciones y su contribución en el desempeño de los trabajadores de la DRA

\begin{tabular}{lccc}
\hline \multicolumn{1}{c}{ Valor cualitativo } & Frecuencia & Porcentaje & $\begin{array}{c}\text { Porcentaje } \\
\text { acumulado }\end{array}$ \\
\hline Totalmente en desacuerdo & 13 & 18 & 18 \\
En desacuerdo & 5 & 7 & 25 \\
Indiferente & 10 & 13 & 38 \\
De acuerdo & 21 & 29 & 67 \\
Totalmente de acuerdo & 24 & 33 & 100 \\
Total & 73 & 100 & \\
\hline \hline
\end{tabular}

Fuente: Cuestionario aplicado a los 73 trabajadores de la Dirección Regional Agraria Amazonas 2019

\subsection{Dirección Regional Agraria y su elaboración del Plan de desarrollo de las personas (PDP).}

Se encontró en la tabla 2 que, el $89 \%$ de los trabajadores consideraron estar totalmente en desacuerdo y en desacuerdo, con la dirección regional agraria ya que no elabora el PDP. El 7\% estuvo de acuerdo, mientras que el $4 \%$ lo indico indiferente.

En otras palabras, se encontró que, no se está cumpliendo la normativa dispuesta por (Autoridad Nacional del Servicio Civil -SERVIR, 2019), establecida por (Decreto Legislativo № 1023, 2008) para la preparación del PDP.

Tabla 2 Dirección Regional Agraria y su elaboración del Plan de desarrollo de las personas.

\begin{tabular}{lccc}
\hline \hline \multicolumn{1}{c}{ Valor Cualitativo } & Frecuencia & Porcentaje & $\begin{array}{c}\text { Porcentaje } \\
\text { acumulado }\end{array}$ \\
\hline Totalmente en desacuerdo & 58 & 79 & 79 \\
En desacuerdo & 7 & 10 & 89 \\
Indiferente & 3 & 4 & 93 \\
De acuerdo & 5 & 7 & 100 \\
Total & 73 & 100 & \\
\hline \hline
\end{tabular}

$\overline{\bar{F} \text { Fuente: Cuestionario aplicado a los } 73 \text { trabajadores de la Dirección Regional Agraria }}$ Amazonas 2019

\subsection{Dimensión de la planificación.}

Se puede apreciar en la tabla 3 que el $81 \%$ de los servidores de la DRA, se encuentran en desacuerdo y totalmente en desacuerdo, en esta dimensión planificación evidenciándose un gran descontento por parte de los trabajadores. El $15 \%$ lo indico indiferente y solo el $4 \%$ estuvo de acuerdo en esta dimensión en términos generales. 
Tabla 3 Dimensión Planificación

\begin{tabular}{lccc}
\hline \hline \multicolumn{1}{c}{ Valor Cualitativo } & Frecuencia & Porcentaje & $\begin{array}{c}\text { Porcentaje } \\
\text { acumulado }\end{array}$ \\
\hline Totalmente en desacuerdo & 14 & 19 & 19 \\
En desacuerdo & 45 & 62 & 81 \\
Indiferente & 11 & 15 & 96 \\
De acuerdo & 3 & 4 & 100 \\
Total & 73 & 100 & \\
\hline \hline
\end{tabular}

Fuente: Cuestionario aplicado a los 73 trabajadores de la Dirección Regional Agraria Amazonas 2019

\subsection{Dimensión de la Ejecución.}

El $86 \%$ de los trabajadores, están en desacuerdo y totalmente en desacuerdo, en esta dimensión ejecución mostrándose insatisfecho por parte de los trabajadores. Mientras que el $10 \%$ lo indico indiferente y solo el $4 \%$ estuvo de acuerdo en esta dimensión en términos generales. Ver tabla 4.

Tabla 4 Dimensión de la Ejecución

\begin{tabular}{lccc}
\hline \hline \multicolumn{1}{c}{ Valor Cualitativo } & Frecuencia & Porcentaje & $\begin{array}{c}\text { Porcentaje } \\
\text { acumulado }\end{array}$ \\
\hline Totalmente en desacuerdo & 44 & 60 & 60 \\
En desacuerdo & 19 & 26 & 86 \\
Indiferente & 7 & 10 & 96 \\
De acuerdo & 2 & 3 & 99 \\
Totalmente de acuerdo & 1 & 1 & 100 \\
Total & 73 & 100 & \\
\hline \hline
\end{tabular}

Fuente: Cuestionario aplicado a los 73 trabajadores de la Dirección Regional Agraria Amazonas 2019

Consecuentemente, se encuentra que, la normativa dispuesta por la (Autoridad Nacional del Servicio Civil -SERVIR, 2019), creada por el (Decreto Legislativo № 1023, 2008), para la ejecución de actividades, no se está cumpliendo.

\subsection{Contenidos de las capacitaciones y su conformidad en las necesidades del cargo.}

El 75\% de los servidores, consideran que los contenidos de las capacitaciones están muy poco y poco acordes a las necesidades de sus cargos. Para el $17 \%$ el contenido es regular, mientras que, para el $8 \%$ el contenido de las capacitaciones es muy acorde. Estos resultados se pueden ver en la tabla 5 . 
Tabla 5 Contenidos de las capacitaciones y su conformidad en las necesidades del cargo.

\begin{tabular}{lccc}
\hline \hline Valor cualitativo & Frecuencia & Porcentaje & $\begin{array}{c}\text { Porcentaje } \\
\text { acumulado }\end{array}$ \\
\hline Muy poco & 35 & 48 & 48 \\
Poco & 20 & 27 & 75 \\
Regular & 12 & 17 & 92 \\
Mucho & 6 & 8 & 100 \\
Total & 73 & 100 & \\
\hline \hline
\end{tabular}

Fuente: Cuestionario aplicado a los 73 trabajadores de la Dirección Regional Agraria Amazonas 2019

\subsection{Técnicas adquiridas en las capacitaciones, y su aplicación en las tareas diarias.}

Para el $77 \%$ de los servidores, en las capacitaciones adquieren pocas técnicas que, posteriormente aplican en sus tareas diarias facilitando su trabajo. Para el $15 \%$ es regular la adquisición de técnicas en las capacitaciones, y solo el $8 \%$ expreso que en las capacitaciones adquieren muchas técnicas.

Tabla 6 Técnicas adquiridas en las capacitaciones, y su aplicación en las tareas diarias.

\begin{tabular}{cccc}
\hline \hline Valor cualitativo & Frecuencia & Porcentaje & $\begin{array}{c}\text { Porcentaje } \\
\text { acumulado }\end{array}$ \\
\hline Muy poco & 39 & 53 & 53 \\
Poco & 17 & 24 & 77 \\
Regular & 11 & 15 & 92 \\
Mucho & 5 & 7 & 99 \\
Bastante & 1 & 1 & 100 \\
Total & 73 & 100 & \\
\hline \hline
\end{tabular}

Fuente: Cuestionario aplicado a los 73 trabajadores de la Dirección Regional Agraria Amazonas 2019

\subsection{Dimensión de la evaluación.}

Se puede notar en la tabla 7 , que el $67 \%$ de los servidores de la DRA-Amazonas, consideraron que se evalúa muy poco, evidenciándose insatisfacción por parte de los trabajadores. Mientras que el $23 \%$ lo indico regular y solo el $9 \%$ consideró que la evaluación se realiza muchas veces. 
Tabla 7 Dimensión de la evaluación

\begin{tabular}{lccc}
\hline \hline Valor cualitativo & Frecuencia & Porcentaje & $\begin{array}{c}\text { Porcentaje } \\
\text { acumulado }\end{array}$ \\
\hline Muy poco & 5 & 7 & 7 \\
Poco & 44 & 60 & 67 \\
Regular & 17 & 23 & 90 \\
Mucho & 6 & 8 & 99 \\
Bastante & 1 & 1 & 100 \\
Total & 73 & 100 & \\
\hline \hline
\end{tabular}

Fuente: Cuestionario aplicado a los 73 trabajadores de la Dirección Regional Agraria Amazonas 2019

2. Servidores de la DRA-Amazonas 2019, y su desempeño

Seguidamente se presenta los resultados concernientes al desempeño de los trabajadores de la DRA, evaluados por los 16 jefes directos.

2.1 Responsabilidad del trabajador en el cumplimiento de los tiempos establecidos.

Según los jefes de la DRA-Amazonas, el $22 \%$ de los trabajadores es deficiente en las responsabilidades para cumplir los tiempos establecidos. El $52 \%$ es regular, mientras que el $26 \%$ es eficiente en las responsabilidades para el cumplimiento de los tiempos establecidos. Ver tabla 8

Tabla 8 Responsabilidad del trabajador en el cumplimiento de los tiempos establecidos.

\begin{tabular}{lccc}
\hline \hline Valor cualitativo & Frecuencia & Porcentaje & $\begin{array}{c}\text { Porcentaje } \\
\text { acumulado }\end{array}$ \\
\hline Deficiente & 16 & 22 & 22 \\
Regular & 38 & 52 & 74 \\
Eficiente & 18 & 25 & 99 \\
Muy Eficiente & 1 & 1 & 100 \\
Total & 73 & 100 & \\
\hline \hline
\end{tabular}

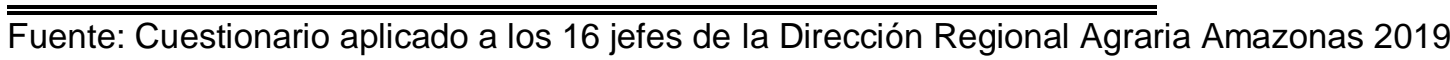

\subsection{Dimensión de la eficiencia.}

Se presenta en la tabla 9 que, el 15\% de los servidores de la DRA-Amazonas, son deficientes en sus áreas de trabajo. Mientras que, el 59\% laboran de manera regular, y el $26 \%$ es eficiente en la Dirección Regional Agraria según lo manifestado por los jefes. 
Tabla 9 Dimensión de la eficiencia

\begin{tabular}{lccc}
\hline Valor cualitativo & Frecuencia & Porcentaje & $\begin{array}{c}\text { Porcentaje } \\
\text { acumulado }\end{array}$ \\
\hline Deficiente & 11 & 15 & 15 \\
Regular & 43 & 59 & 74 \\
Eficiente & 18 & 25 & 99 \\
Muy Eficiente & 1 & 1 & 100 \\
Total & 73 & 100 & \\
\hline \hline
\end{tabular}

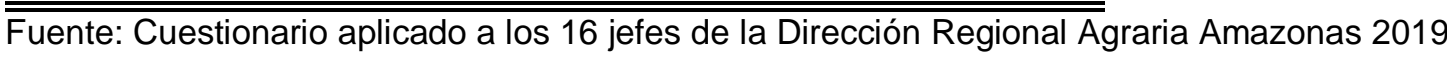

\subsection{Dimensión de la eficacia.}

Respecto a la eficacia, el $8 \%$ de los servidores de la DRA, se desempeña de manera ineficaz. Sin embargo, el $62 \%$ de los trabajadores se desempeñan de manera regular, y el $30 \%$ son eficaces en sus puestos de trabajos. Ver tabla 10

Tabla 10 Dimensión de la eficacia

\begin{tabular}{cccc}
\hline \hline Valor cualitativo & Frecuencia & Porcentaje & $\begin{array}{c}\text { Porcentaje } \\
\text { acumulado }\end{array}$ \\
\hline Ineficaz & 6 & 8 & 8 \\
Regular & 45 & 62 & 70 \\
Eficaz & 22 & 30 & 100 \\
Total & 73 & 100 & \\
\hline \hline
\end{tabular}

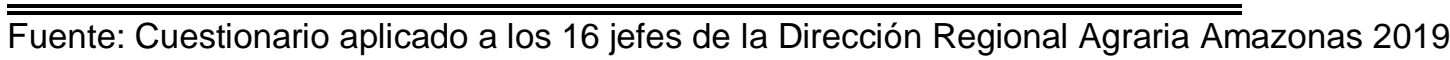

\subsection{Desempeño de los servidores de la DRA-Amazonas 2019.}

Respecto al desempeño laboral de los trabajadores, según lo manifestado por sus jefes directos en la DRA-Amazonas, se nota en la tabla 11 que, el $8 \%$ de los trabajadores, son deficientes e ineficaces. El $62 \%$ es Regular en el desempeño, mientras que solo el $30 \%$ de los trabajadores son eficientes y eficaces.

\begin{tabular}{lccc} 
Tabla 11 Desempeño Laboral & & \\
\hline \hline Valor Cualitativo & Frecuencia & Porcentaje & $\begin{array}{c}\text { Porcentaje } \\
\text { acumulado }\end{array}$ \\
\hline Deficiente/Ineficaz & 6 & 8 & 8 \\
Regular & 45 & 62 & 70 \\
Eficiente/Eficaz & 22 & 30 & 100 \\
Total & 73 & 100 & \\
\hline \hline
\end{tabular}

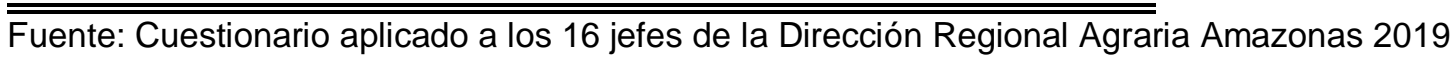




\section{Asociaciones entre las actividades del adiestramiento y el desempeño de los servidores de la DRA-Amazonas 2019.}

Líneas abajo se aprecian los resultados sobre las relaciones concernientes a las actividades capacitación conformadas por: Planeación, Ejecución, y Evaluación, y el desempeño laboral evaluado por los 16 jefes directos de los servidores de la DRAAmazonas.

3.1 Relación entre las actividades de planificación del adiestramiento y el desempeño de los servidores de la DRA-Amazonas 2019.

La planificación del adiestramiento, se encuentra relacionada con el promedio del desempeño, por haberse encontrado una relación positiva moderada, con una correlación de 0,50.

Tabla 12 Relación entre la planificación de la capacitación, el desempeño laboral de los trabajadores de la Dirección Regional Agraria Amazonas.

\begin{tabular}{llc}
\hline \hline & & $\begin{array}{c}\text { Desempeño } \\
\text { Laboral }\end{array}$ \\
\hline \multirow{3}{*}{ Planificación } & Correlación de Pearson &, $502^{* *}$ \\
& Sig. (bilateral) &, 000 \\
& $\mathrm{~N}$ & 73
\end{tabular}

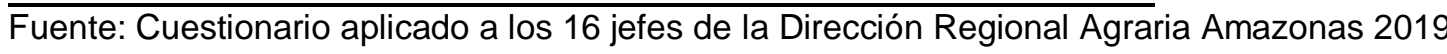

\subsection{Relación de las actividades de ejecución del adiestramiento y el desempeño} de los servidores de la DRE-Amazonas 2019.

La ejecución del adiestramiento, se encuentra relacionada con el promedio del desempeño, por hallarse una correlación de 0,58.

Tabla 13 Relación entre la ejecución de la capacitación, y el desempeño laboral de los trabajadores de la Dirección Regional Agraria Amazonas

\begin{tabular}{llc}
\hline \hline & & $\begin{array}{c}\text { Desempeño } \\
\text { Laboral }\end{array}$ \\
\hline \multirow{3}{*}{ Ejecución } & Sig. (bilateral) &, $584^{* *}$ \\
& $\mathrm{~N}$ &, 000 \\
& & 73 \\
\hline \hline
\end{tabular}

$\overline{\overline{F u e n t e: ~ C u e s t i o n a r i o ~ a p l i c a d o ~ a ~ l o s ~} 16 \text { jefes de la Dirección Regional }}$ Agraria Amazonas 2019 Elaboración: Propia 
3.3 Relación entre las actividades de evaluación del adiestramiento y el desempeño de los servidores de la DRA-Amazonas 2019.

La evaluación del adiestramiento se relaciona con el promedio del desempeño de los servidores, al encontrarse una correlación de Pearson de 0,495.

Tabla 14 Relación entre la evaluación de la capacitación, y el desempeño laboral de los trabajadores de la Dirección Regional Agraria Amazonas

\begin{tabular}{llc}
\hline \hline \multirow{4}{*}{ Evaluación } & Correlación de Pearson & $\begin{array}{c}\text { Desempeño } \\
\text { Laboral }\end{array}$ \\
& Sig. (bilateral) &, $495^{\star *}$ \\
& $\mathrm{~N}$ &, 000 \\
& & 73
\end{tabular}

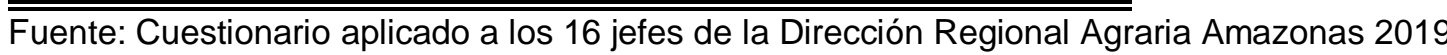
Elaboración: Propia

\section{DISCUSIÓN}

El $62 \%$ de los servidores están de acuerdo que el adiestramiento contribuye en el desempeño de los servidores de la DRA. El 25\% de los encuestados manifestó estar en desacuerdo, mientras que solo el $13 \%$ se mostró indiferente. De manera similar (Mayurí, 2008), puesto que, en ambas investigaciones, más del 60\% está de acuerdo en que, las capacitaciones contribuyen al mejor desempeño de los trabajadores.

El $75 \%$ de los entrevistados, consideran que el tema de las capacitaciones está poco relacionado con las necesidades de sus cargos. El 17\% manifiestan regular el contenido de las capacitaciones, mientras que el $8 \%$ lo indicó muy acordes. De manera contraria se observó en la investigación realizada por (Alvarado, Gonzalez, Lopez, \& Perez, 2002), se aprecia que: "El 93.33\% de los empleados, consideran que los temas de la última capacitación estaban acordes a las necesidades de su puesto, sólo el $6.67 \%$ considera que no están acorde los contenidos".

Se pueden apreciar resultados completamente opuesto, siendo que, en la DRAAmazonas, para el $75 \%$ de los servidores, el contenido de las capacitaciones no está acorde a las necesidades de sus cargos, y en lo encontrado por (Alvarado, Gonzalez, Lopez, \& Perez, 2002), solo el 6,67\% indica que el contenido no es acorde, existiendo una diferencia porcentual de 68 puntos.

Para el $77 \%$ de los servidores, en las capacitaciones adquieren pocas técnicas que, posteriormente aplican en sus tareas diarias facilitando su trabajo. El 15\% lo manifestó regular la adquisición de técnicas en las capacitaciones, y solo el $8 \%$ expreso que las aplican mucho. Observando gran diferencia con la investigación realizada por (Noda, 2017), quien observó que: el 100\% de los servidores, entre siempre y frecuentemente, 
alcanzan técnicas facilitándole el trabajo. A diferencia del $8 \%$ de los servidores de la DRA-Amazonas que, adquiere técnicas que posteriormente aplican en su trabajo.

El $22 \%$ de los jefes directos de los trabajadores manifestaron que la responsabilidad del trabajador en cumplir los tiempos establecidos es deficiente. El 52\% indicó que es regular, mientras que el $26 \%$ lo evaluó eficiente. Encontrándose semejanzas con la investigación realizada por (Colque, 2016), quien encontró que: "El desempeño laboral no es el esperado, en el caso de la responsabilidad, para el $43 \%$ es desfavorable, para el $29 \%$ es favorable, y muy favorable, mientras que para el $28 \%$, la responsabilidad, se desarrolla en nivel medio"

Las semejanzas encontradas se pueden apreciar en el porcentaje que encontró favorable y eficiente el cumplimiento de la responsabilidad, siendo para la presente investigación el 26\%, y en lo encontrado por (Colque, 2016), para el 29\% es favorable. El $8 \%$ de los jefes directos, manifestaron que los trabajadores son deficientes e ineficaces. Para el $62 \%$ el desempeño es regular, mientras que solo el $30 \%$ considero que son eficientes y eficaces, en el desempeño laboral. Encontrándose diferencias con (Rengifo, 2018), en su investigación se observó que: El desempeño laboral para el $18,5 \%$ de los trabajadores, se encuentra en un nivel alto.

Las diferencias encontradas se destacan en el $30 \%$ que considera que los servidores de la DRA-Amazonas, son eficientes y eficaces, en el desempeño laboral, mientras que, según (Rengifo, 2018), solo el 18,5\% presenta un alto nivel en el desempeño laboral.

\section{CONCLUSIONES.}

Primera: Las actividades de adiestramiento se relacionan de manera moderada con el desempeño de los trabajadores de la DRA. Sin embargo, a pesar de que los trabajadores no reciben la adecuada capacitación, incumpliéndose las disposiciones (art. 10) del Decreto Legislativo $N^{\circ} 1023$; su desempeño laboral se desarrolla de manera regular en la DRA.

Segunda: Existe un descontento de parte de los trabajadores en cuanto a la planificación de la capacitación, puesto que, el $81 \%$ de ellos están en desacuerdo, con la actividad de planificación de la capacitación, es decir, no se está cumpliendo con el acápite a del (Decreto Legislativo № 1023, 2008, pág. 3)

Tercera: El 86\% de los servidores de la DRA, expresaron estar en desacuerdo, con forma como ejecutan la capacitación. Encontrándose correlación de 0,58, con el desempeño de los servidores. 
Cuarta: Se evidencia descontento en la evaluación de la capacitación, por parte de los trabajadores, puesto que para el $67 \%$ de los servidores de la DRA, la evaluación se realiza muy poco. Se encontró una correlación de Pearson de 0,495 , lo que quiere decir que, la evaluación influye en el desempeño de los servidores, es decir, hay una relación positiva, entre estos elementos.

Quinta: La DRA-Amazonas, no se está cumpliendo con lo dispuesto en el Convenio 142, dispuesto por la (Organización Internacional del Trabajo, 1975), en cuanto a la profesionalización de los servidores.

Sexta: El incumplimiento del (Decreto Legislativo № 1023, 2008, pág. 3), está influyendo relativamente, en el desempeño de los servidores de la DRA.

Finalmente, se recomienda que, se formalicen convenios con el Gobierno Regional, a fin de que, se puedan realizar capacitaciones en la Dirección Regional Agraria de Amazonas, a través de la Universidad Nacional Toribio Rodríguez de Mendoza de Amazonas, a quien el Gobierno Regional le financia grandes presupuestos para la ejecución de sus proyectos.

\section{REFERENCIAS BIBLIOGRÁFICAS.}

Alvarado, N., Gonzalez, J., Lopez, G., \& Perez, A. (2002). "La capacitación y su incidencia en el desempeño laboral de empleados de instituciones públicas y privadas; y propuesta de un manual de capacitación”. El salvador. Obtenido de http://ri.ues.edu.sv/id/eprint/10113/1/14100308.pdf

Autoridad Nacional del Servicio Civil. (8 de 8 de 2016). Normas para la gestión del proceso de capacitación en las entidades públicas. Obtenido de https://storage.servir.gob.pe:

https://storage.servir.gob.pe/normatividad/Resoluciones/PE-2016/Res1412016-SERVIR-PE.pdf

Autoridad Nacional del Servicio Civil. (2019). Servicio Civil . Obtenido de Autoridad Nacional del Servicio Civil -SERVIR: https://www.servir.gob.pe/servicio-civil/

Autoridad Nacional del Servicio Civil -SERVIR. (2019). Gestión de Capacitación,Gobiernos Regionales. Obtenido de www.servir.gob.pe: https://www.servir.gob.pe/gdc/entidades/pdp-gobiernos-regionales/

Bonnefoy, J. (2005). Indicadores de desempeño en el sector público. Santiago de Chile. Recuperado el 21 de 02 de 2019, de https://repositorio.cepal.org/bitstream/handle/11362/5611/S05900 es.pdf

Chiavenato, I. (2009). Gestión de Talento Humano (Tercera edición ed.). México: McGRAW-HILL/INTERAMERICANA EDITORES, S.A. Recuperado el 27 de 02 
de 2019, de http://www.facso.unsj.edu.ar/catedras/cienciaseconomicas/administracion-de-personal-I/documentos/chiavena.pdf

Colque, C. (2016). Influencia de la capacitación institucional en el desempeño laboral de los trabajadores de los centros de investigación y producción de la Universidad Nacional del Altiplano - 2011. Puno-Perú. Recuperado el 27 de 02 de 2019 , de

http://repositorio.unap.edu.pe/bitstream/handle/UNAP/6612/EPG966-00966-

01. pdf? sequence $=1$ \&isAllowed $=\mathrm{y}$

Decreto Legislativo № 1023. (2008). DECRETO LEGISLATIVO QUE CREA LA AUTORIDAD NACIONAL DEL SERVICIO CIVIL, RECTORA DEL SISTEMA ADMINISTRATIVO DE GESTIÓN DE RECURSOS HUMANOS. . Lima.

Obtenido de http://www.oas.org/juridico/PDFs/mesicic5 per 4 dec leg 1023.p Decreto Supremo № 009-2010-PCM. (2010). Aprueban el Reglamento del Decreto Legislativo № 1025 sobre Normas de Capacitación y Rendimiento para el Sector Público. Lima. Obtenido de http://www2.pcm.gob.pe/Transparencia/Resol ministeriales/2010/DS-0092010-

Días, R. (2011). Capacitación y desempeño laboral de los empleados de la comisión federal de electricidad de la Zona Montemorelos-Linares Nuevo León. México. Obtenido de http://dspace.biblioteca.um.edu.mx/xmlui/handle/20.500.11972/88

Fuentes, I. (2014). Plan de Capacitación y el desempeño laboral de personal del proyecto especial Chavimochic en el año 2012. Trujillo. Obtenido de http://dspace.unitru.edu.pe/handle/UNITRU/963

Jamaica, F. (2015). Los beneficios de la capacitación y el desarrollo del personal de las pequeñas empresas. BOGOTÁ. Recuperado el 19 de 02 de 2019, de https://repository.unimilitar.edu.co/bitstream/handle/10654/7168/Trabajo\%20fin al\%20Fabian\%20Jamaica\%20(1).pdf?sequence=1

Lacavex, M. (2013). Capacitación y adiestramiento para el trabajo en el Derecho Mexicano. Revista de Derecho de la Pontificia Universidad Católica de Valparaíso(41). doi:doi:http://dx.doi.org/10.4067/S0718-68512013000200003

Mayurí, J. (2008). Capacitación empresarial y desempeño laboral en el Fondo de Empleados del Banco de La Nación FEBAN, Lima 2006. Lima. Obtenido de http://cybertesis.unmsm.edu.pe/handle/cybertesis/2615

Ministerio de Economía y Finanzas. (2010). Instructivo para la Formulación de Indicadores. DIRECCIÓN GENERAL DEL PRESUPUESTO PÚBLICO, Lima. $\begin{array}{lllll}\text { Recuperado el de } 20 \text { de } & \end{array}$ 
https://www.mef.gob.pe/contenidos/presupuesto publico/normativa/Instructivo Formulacion Indicadores Desempeno.pdf

Noda, S. (2017). Capacitación y el desempeño laboral de los trabajadores del Restaurante de Mar y Piedra de la Ciudad de Huánuco 2017. Huánuco - Perú. Obtenido de http://repositorio.udh.edu.pe/bitstream/handle/123456789/813/T047 47909453 T.pdf?sequence $=1 \& \mathrm{i}$

Obregón, M., Alonso, B., Díaz, V., Iglesias, F., Rodríguez, L., Fernández, D., . . Mevil, J. (2008). Capacitación y desarrollo de los Recursos Humanos. Manual, MINISTERIO DE SALUD PÚBLICA, Habana. Recuperado el 19 de 02 de 2019, de http://www.sld.cu/galerias/pdf/sitios/cpich/manual de capacitacion. tomo ii.pdf

OIT - Lima. (2019). Organización Internacional del Trabajo. Lima. Obtenido de https://www.ilo.org/lima/paises/per\%C3\%BA/lang--es/index.htm

Organización Internacional del Trabajo. (1975). C142 - Convenio sobre el desarrollo de los recursos humanos. Obtenido de https://www.ilo.org/dyn/normlex/es/f?p=NORMLEXPUB:12100:0::NO::P12100 | LO CODE:C142

Parra-Penagos, C., \& Rodríguez-Fonseca, F. (Enero - Junio de 2016). La capacitación y su efecto en la calidad dentro de las organizaciones. 131Rev. Investig. Desarro. Innov, 6(2), 131-143. Obtenido de http://dx.doi.org/10.19053/20278306.4602

Qian, X., \& Zhou, L. (2008). Modelo de formación y desarrollo a implementar en las Universidades. Universidad Central de las Villa. Recuperado el 19 de 02 de 2019, de http://www.eumed.net/libros-gratis/2008b/406/index.htm

Ramírez, A. (2014). Capacitación y desempeño laboral de los empleados de la Universidad Linda Vista de la Unión Mexicana de Chiapas. México. Obtenido de http://dspace.biblioteca.um.edu.mx/xmlui/handle/20.500.11972/98

Rengifo, R. (2018). Proceso de capacitación y el desempeño laboral del personal administrativo del organismo de evaluación y fiscalización ambiental, Lima 2017. Lima. Obtenido de http://repositorio.ucv.edu.pe/bitstream/handle/UCV/16316/Rengifo MRM.pdf?s eq 